\title{
Channeling through Bent Crystals
}

\author{
Stephanie Mack \\ Office of Science, Science Undergraduate Laboratory Internship (SULI) \\ University of Ottawa \\ SLAC National Accelerator Laboratory \\ Menlo Park, CA
}

August 19, 2011

Prepared in partial fulfillment of the requirements of the Office of Science, Department of Energy's Science Undergraduate Laboratory Internship under the direction of H.-Ulrich (Uli) Wienands at the Stanford University, SLAC National Accelerator Laboratory.

Participant:

Signature

Research Advisor:

Signature 


\section{TABLE OF CONTENTS}

$\begin{array}{ll}\text { Abstract } & \text { ii }\end{array}$

$\begin{array}{ll}\text { Introduction } & 1\end{array}$

Materials and Methods 2

$\begin{array}{ll}\text { Results } & 3\end{array}$

$\begin{array}{ll}\text { Discussion and Conclusions } & 5\end{array}$

$\begin{array}{lr}\text { Acknowledgments } & 7\end{array}$ 


\begin{abstract}
Channeling through Bent Crystals. STEPHANIE MACK (University of Ottawa, Ottawa, ON K1N 6N5), H.-ULRICH (ULI) WIENANDS (Stanford University, SLAC National Accelerator Laboratory, Menlo Park, CA 94025).
\end{abstract}

Bent crystals have demonstrated potential for use in beam collimation. A process called channeling is when accelerated particle beams are trapped by the nuclear potentials in the atomic planes within a crystal lattice. If the crystal is bent then the particles can follow the bending angle of the crystal. There are several different effects that are observed when particles travel through a bent crystal including dechanneling, volume capture, volume reflection and channeling. With a crystal placed at the edge of a particle beam, part of the fringe of the beam can be deflected away towards a detector or beam dump, thus helping collimate the beam. There is currently FORTRAN code by Igor Yazynin that has been used to model the passage of particles through a bent crystal. Using this code, the effects mentioned were explored for beam energy that would be seen at the Facility for Advanced Accelerator Experimental Tests (FACET) at a range of crystal orientations with respect to the incoming beam. After propagating 5 meters in vacuum space past the crystal the channeled particles were observed to separate from most of the beam with some noise due to dechanneled particles. Progressively smaller bending radii, with corresponding shorter crystal lengths, were compared and it was seen that multiple scattering decreases with the length of the crystal therefore allowing for cleaner detection of the channeled particles. The input beam was then modified and only a portion of the beam sent through the crystal. With the majority of the beam not affected by the crystal, most particles were not deflected and after propagation the channeled particles were seen to be deflected approximately $5 \mathrm{~mm}$. After a portion of the beam travels through the crystal, the entire beam was then sent through a quadrupole magnet, which increased the separation of the channeled particles 
from the remainder of the beam to a distance of around $20 \mathrm{~mm}$. A different code, which was developed at SLAC, was used to create an angular profile plot which was compared to what was produced by Yazynin's code for a beam with no multiple scattering. The results were comparable, with volume reflection and channeling effects observed and the range of crystal orientations at which volume reflection is seen was about $1 \mathrm{mrad}$ in both simulations. 


\section{INTRODUCTION}

Crystals contain atoms arranged in a lattice which forms planes. When accelerated particles enter a crystal parallel to a plane they can be trapped by the nuclear potentials, a process called channeling [1]. If the crystal is bent elastically then particles may follow the curvature of the planes. This concept can then be applied to beam collimation: if a crystal is placed to intercept the halo particles of a beam these can be deflected away from the beam to a detector or beam dump. This may improve the collimation of the beam which is important for preventing damage to equipment and improving the noise to signal ratio of experiments using the beam. Previous channeling experiments have examined this effect at circular accelerators including the RD22 experiment at the H8 beamline at the European Organization for Nuclear Research (CERN) [2] and the Tevatron at Fermilab [3].

An experiment to be proposed for the Facility for Advanced Accelerator Experimental Tests (FACET) at SLAC National Accelerator Laboratory (SLAC) will study the effectiveness of a bent crystal for collimation of the $23 \mathrm{GeV}$ positron/electron beam using planar channeling with a silicon (110) crystal. The CRY_AP code written in Fortran by Igor Yazynin and modified by Robert J. Noble [4] provides the baseline for the study of the bent crystal. The effects of volume reflection, volume capture, channeling and dechanneling were investigated with a beam of positively charged particles passing through the crystal. Volume capture happens when a particle is not initially channeled, but as it passes through successive planes it eventually makes a critical angle with a plane and becomes channeled. A dechanneled particle is one that is initially channeled between the planes but then exits. These processes are largely caused by multiple scattering of the particle. Volume reflection occurs when the particles hit the planes of the crystal at a shallow angle. They are thus reflected off the plane and do not get trapped between the planes and are only slightly deviated from a straight path.

The crystal parameters were optimized for FACET beam energy. A more realistic input 
beam was then prepared and the crystal placed to intercept the edge of the beam. The effectiveness of channeling after passing through the crystal and propagating a certain distance to a detector was investigated. Beyond the crystal a quadrupole magnet was then added to the code to improve separation of channeled particles from the remainder of the beam to improve the experimental setup. Another code written at SLAC [5], which employs a different method of computation for determining the particles' positions and angles, was then compared to the results of Yazynin's code. This work focuses on channeling of positrons as the process is less efficient with electrons due to their being attracted by the nuclei.

\section{MATERIALS AND METHODS}

The main tool used for analysis of positively charged particles passing through a bent crystal was the FORTRAN code CRY_AP written by Igor Yazynin and modified by Robert J. Noble [4]. The code uses a truncated Monte Carlo method for determining the position and angle of the particle as it exits the crystal. It includes the volume reflection, volume capture, dechanneling and channeling effects. Nuclear and multiple scattering are also taken into account.

This code was modified to make a more realistic comparison to experimental conditions in FACET. The divergence of the beam was added in to the initialization of the position of the particles. A drift space was added so that after passing through the crystal, the particles propagated five meters and their angle and position were calculated at that point to determine what a detector would measure. Furthermore, instead of initializing the particles with a random number generator, a datafile was constructed with an initial position and angle assigned to each particle with a phase space ellipse similar to what would be expected in the accelerator. This input beam was then implemented into the code and positioned so only the edge of the beam was intercepted by the crystal. These particles were also propagated in vacuum space. 
In FACET it would be possible to propagate the particles further from the crystal in order to allow more separation between the main beam and the channeled portion, but they would pass through a quadrupole magnet. Therefore the code was adjusted to take into account the manipulation of the beam due to the magnet. The transform matrix was obtained from a magnet-lattice model of FACET.

The relevant FACET parameters were calculated for positrons and are included in Table 1. As well, the critical angle for channeling in silicon (110) crystals was calculated using the formula

$$
\Theta_{c}=\sqrt{\frac{2 * U_{\max }}{p v}}\left(1-\frac{R_{T}}{R_{m}}\right)
$$

where $\mathrm{U}_{\max }$ is the maximum potential energy, $\mathrm{p}$ is the momentum, $\mathrm{v}$ is the velocity, $\mathrm{R}_{T}$ is the Tsyganov radius and $\mathrm{R}_{m}$ is the bending radius. The critical angle was found to be 42.6 $\mu \mathrm{rad}$.

The second code performs detailed tracking to decide the particle's angle and position. It uses many steps through the crystal and averages the atomic potential along the planes according to the Lindhard Potential [1]. This code does not calculate multiple scattering effects on the particles.

\section{RESULTS}

The angular profile plot for the crystal was used primarily for the analysis. The deflection of the particles as a function of the crystal orientation, the angle the crystal planes make with the incoming beam, is plotted. For a $10 \mathrm{~mm}$ long crystal with a $10 \mathrm{~m}$ bending radius with multiple scattering effects turned off in the code, a basic view of the amorphous region, volume reflection, and channeled particles is outlined in green in Fig. 1. When multiple scattering is included, the beam width increases significantly as seen by the red data points, and the volume capture and dechanneled particles are clearly observed.

Upon implementing the propagation of the particles to a detector, a histogram was 
created in order to see the distribution of the particles. For the amorphous region, in Fig. 2 the histogram shows a peak of particles centered around the middle, with no displacement, with a certain width of the distribution of the beam due to scattering. Fig. 3 illustrates a slice of the crystal orientation which includes volume reflection and volume capture, seen as two clearly defined peaks of intensity. In the channeling orientation of the crystal, the channeled particles have the highest intensity relative to the rest of the beam (Fig. 4).

In order to optimize the crystal parameters, the same analysis was done for three different bending radii and crystal lengths but the same overall bending angle. In Fig. 5 is shown the angular plots overlaid for a $10 \mathrm{~m} / 10 \mathrm{~mm}, 5 \mathrm{~m} / 5 \mathrm{~mm}, 2.5 \mathrm{~m} / 2.5 \mathrm{~mm}$ bending radius/crystal length. These particles were also propagated $5 \mathrm{~m}$ after passing through the crystal and their distribution is plotted in Fig. 6.

A phase space plot of the new input beam is shown in Fig. 7. This beam was offset so that only a portion would pass through the crystal, which was placed at the zero position, and the rest of the beam would not be affected by it. The portion traveling through the crystal is highlighted in green. Fig. 8 shows the same phase space plot of the particles at the exit face of the crystal, for a crystal of a $5 \mathrm{~m}$ bending radius and a length of $5 \mathrm{~mm}$. The distribution of particles is plotted in Fig. 9 upon propagating $5 \mathrm{~m}$. Then in place of just propagating $5 \mathrm{~m}$ in vacuum space, after the crystal the entire beam was sent through a quadrupole magnet (analogous to the what is in place in the beamline at FACET) and the distribution of the particles after the magnet is plotted in Fig. 10.

Using the code developed at SLAC [5], the result of the angular profile was compared to what was observed with Yazynin's code. The angular profile plot in Fig. 11 demonstrates that there are strong similarities; it should be noted that the vertical line parallel to the main beam in the figure is an artifact of the code dependent on the number of steps taken. 


\section{DISCUSSION AND CONCLUSIONS}

The angular profile plots match the overall form expected from previous research [2] with the volume reflection, capture, dechanneling and channeling effects taken into account in the code. Comparing the angular profiles in Fig. 1, the effect of multiple scattering is easily seen. The beam width increases significantly from a narrow beam without the multiple scattering. As well, the volume capture effect and dechanneling are dependent on scattering. The scattered particles far from the main part of the beam (i.e. when the absolute value of the deflection is greater than approximately $0.5 \mathrm{mrad}$ ) are largely due to nuclear scattering and large angle multiple scattering.

Using this angular profile to determine the orientation of the crystal to observe different channeling effects, the distribution of particles upon propagating $5 \mathrm{~m}$ past the crystal was investigated using a crystal with a bending radius of $10 \mathrm{~m}$ and a length of $10 \mathrm{~mm}$. In the amorphous region, where the beam interacts minimally, the distribution in Fig. 2 shows a single peak with a certain width which can be attributed to the scattering effects. Upon changing the orientation of the crystal to observe volume capture and volume reflection in Fig. 3, the distribution changes significantly. The wider peak on the left representing the volume reflected portion of the beam illustrates that it would be difficult to use a detector to observe this effect since the displacement of these particles from zero is minimal compared to the width of the peak. There is some noise between the two peaks which reduces the clarity of the peaks. The volume captured particles have a higher intensity with a narrower displacement distribution than the volume reflected particles.

At channeling orientation of the crystal, channeling of the particles is easily detected in Fig. 4. There is a small peak in the distribution where the beam is minimally deflected. The intensity of the channeled particles is observed around a deflection of $5 \mathrm{~mm}$. However, there is significant noise between these two peaks due to the dechanneled particles. In order to minimize this effect and optimize channeling, different bending radii and crystal lengths 
were compared. As observed in Fig. 5 the beam width (the amount of multiple scattering) decreases with crystal length. As well, the density of dechanneled particles can be seen to be less with the $2.5 \mathrm{~m}$ bending radius than the $5 \mathrm{~m}$ or $10 \mathrm{~m}$ bending radius since the particles are less dense in that area on the plot. When using Fig. 5 and Fig. 6 to compare the different bending radii, one can observe the decreased multiple scattering with a decrease in the bending radius. The intensity of channeled particles almost doubles as the bending radius goes down by a factor of four and the angle of deflection for these particles remains constant. The noise due to dechanneled particles between the two peaks does decrease with the shorter bending radius. This would improve the ability to cleanly detect the channeled particles in an experimental setup. However, mechanical constraints will hinder the feasibility of attaining a crystal with a very small bending radius. Therefore, the remaining simulations used a $5 \mathrm{~mm}$ long crystal with a $5 \mathrm{~m}$ bending radius.

The code originally initializes the particles with a fixed position and angle then adds a randomized element using a random number generator. To better simulate the beam that would be in FACET, a data file with particles assigned positions and angles that make a phase space ellipse as seen in Fig. 7 was created. Instead of passing the entire beam through the crystal, since the crystal would be used for collimation, the beam was offset from the crystal so only a portion of the beam actually passes through as illustrated with the green portion of the beam. At the exit face of the crystal the main part of the beam remains unchanged but the portion going through the crystal is subject to dechanneling, channeling, volume capture/reflection as is illustrated in Fig. 8. One can see that of the part of the beam going through the crystal, the particles are mostly either not deflected, volume reflected or channeled with some dechanneling occurring. Since the majority of the beam is not passed through the crystal there is a high intensity of particles that are not deflected in Fig. 9. The channeled particles represent less than $5 \%$ of the beam but are still detectable at a deflection of approximately $5 \mathrm{~mm}$.

Beyond where the crystal would be placed in FACET is a quadrupole magnet. This would 
affect the beam and so using the input beam in Fig. 7, again with the portion highlighted in green actually going to the crystal, the entire beam was then passed through the magnet and the particle distribution plotted in Fig. 10. Similar to Fig. 9, the majority of the beam is unaffected causing a large peak close to zero. The channeled particles are deflected approximately $20 \mathrm{~mm}$ with less than $5 \%$ channeled.

A comparison was made between the angular profile of a beam with no multiple scattering, with the entire beam going through the crystal, and the deflection as a function of crystal orientation between the code developed at SLAC (Fig. 11) and Yazynin's code. The volume reflection and channeling can be easily observed. The range of orientations of the crystal for which volume reflection occurs is approximately $1 \mathrm{mrad}$, comparable to the range seen in Fig. 1. Further modifications need to be made to the code, including removing the artifact vertical band parallel to the beam and possibly including multiple scattering effects.

Bent crystals have applications for beam collimation. A particle beam can be passed through a crystal and depending on the orientation of the crystal, various effects can be observed. A shorter bending radius along with a shorter length of crystal improved the detection of channeled particles after they have propagated $5 \mathrm{~m}$ past the crystal. Propagating the particles through the quadrupole magnet increases the distance the channeled particles are deflected. The placement of a detector could also be investigated further to improve the experimental setup. As well, the code could be modified to include negatively charged particles. It appears that a bent crystal could be used to channel a portion of the positron beam in FACET.

\section{ACKNOWLEDGMENTS}

This research was made possible by the United States Department of Energy Summer Undergraduate Laboratory Internship (SULI). I would like to thank SLAC National Accelerator Laboratory and Stanford University for hosting me this summer. Lastly I would like 
to thank Uli Wienands for providing me with this interesting project and mentoring me throughout my time at SLAC.

\section{REFERENCES}

[1] S. Møller "High-energy channeling - applications in beam bending and extraction". Nucl. Instrum. Meth. A, vol. 361, no. 3, 403-420, July 1995

[2] W. Scandale, "High-Efficiency Volume Reflection of an Ultrarelativistic Proton Beam with a Bent Silicon Crystal," Phys. Rev.Lett., vol. 98, no. 15, 154801, Apr. 2007

[3] R.A. Carrigan, "Beam extraction studies at $900 \mathrm{GeV}$ using a channeling crystal". Phys. Rev. ST Accel. Beams, vol. 5, no. 4, 043501, Apr. 2002

[4] R.J. Noble, "Proton-Nucleus Scattering Approximation and Implications for LHC Crystal Collimation," Nucl. Instrum. Meth. A, vol. 623, no. 3, 872-882, Nov. 2010

[5] U. Wienands, SLAC National Accelerator Laboratory, private communication 


\section{FIGURES}

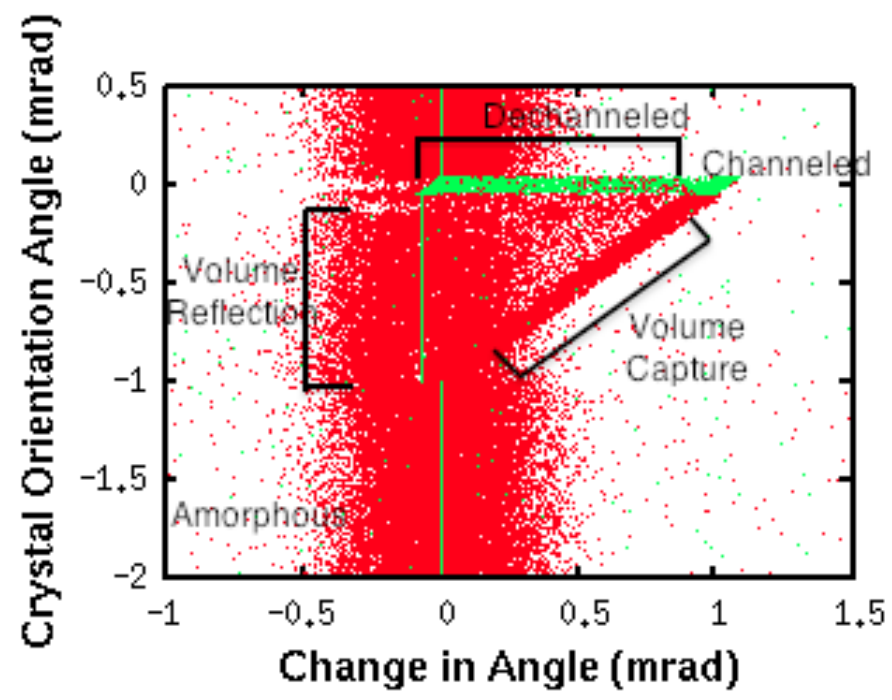

Figure 1: Angular profile of the crystal representing the angular deflection of the particles as a function of the crystal orientation. The red points represent when multiple scattering is included and the green points are when it is not included in the calculations. The crystal in $10 \mathrm{~mm}$ in length and has a $10 \mathrm{~m}$ bending radius. The labels indicate the effects that occur at various crystal orientations. The crystal is aligned with the beam at an orientation angle of 0 .

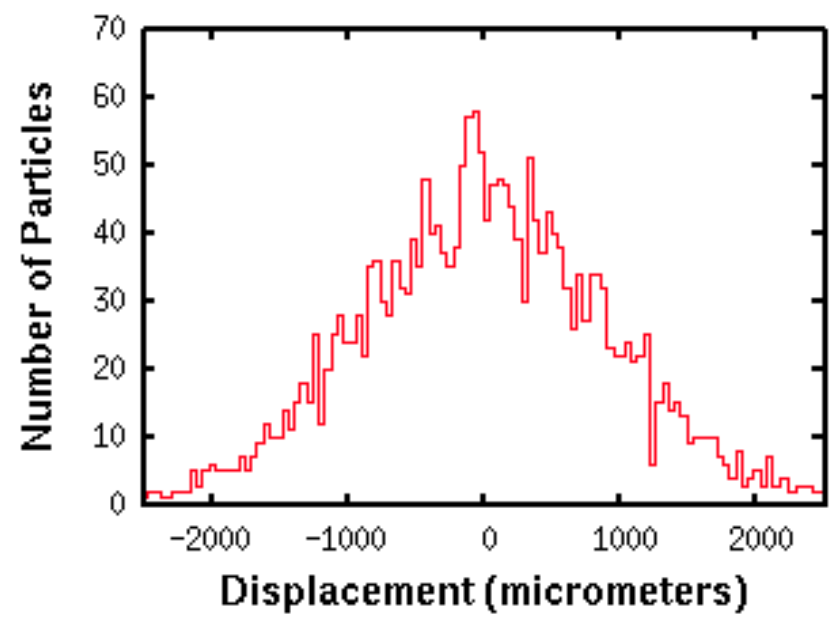

Figure 2: Histogram of the displacement of particles after propagating five meters beyond the crystal in vacuum space. The orientation angle of the crystal is from $0.2 \mathrm{mrad}$ to $0.3 \mathrm{mrad}$ representing the amorphous region. 


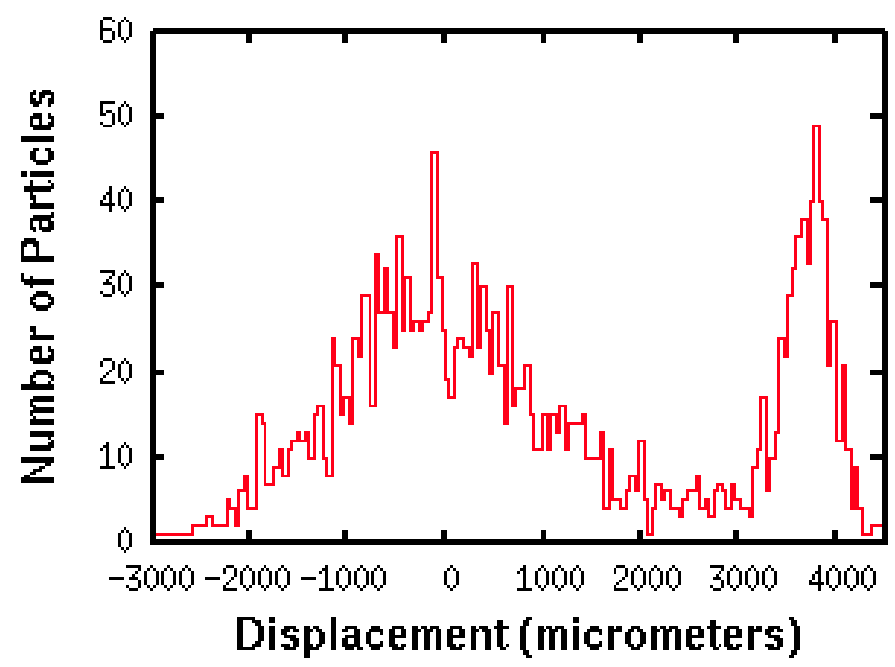

Figure 3: Histogram of the displacement of particles after propagating five meters beyond the crystal in vacuum space. The orientation angle of the crystal is from $-0.3 \mathrm{mrad}$ to $-0.2 \mathrm{mrad}$ representing a region with volume reflection (peak on the left) and volume capture (peak on the right).

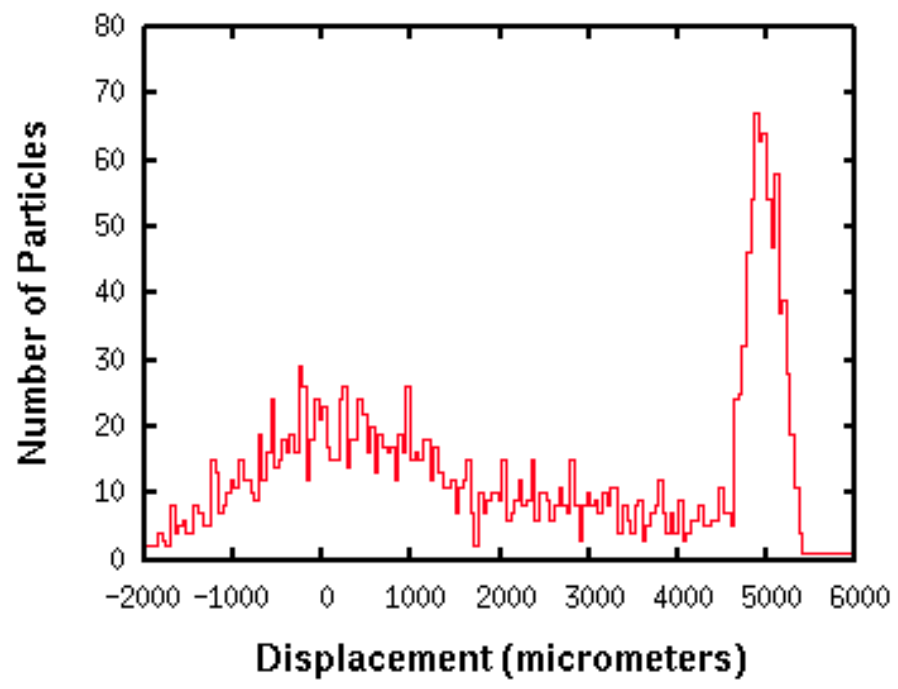

Figure 4: Histogram of the displacement of particles after propagating five meters beyond the crystal in vacuum space. The orientation angle of the crystal is from $-0.051 \mathrm{mrad}$ to $0.049 \mathrm{mrad}$. The peak on the right is channeled particles and the unaffected particles are centered around 0 . 


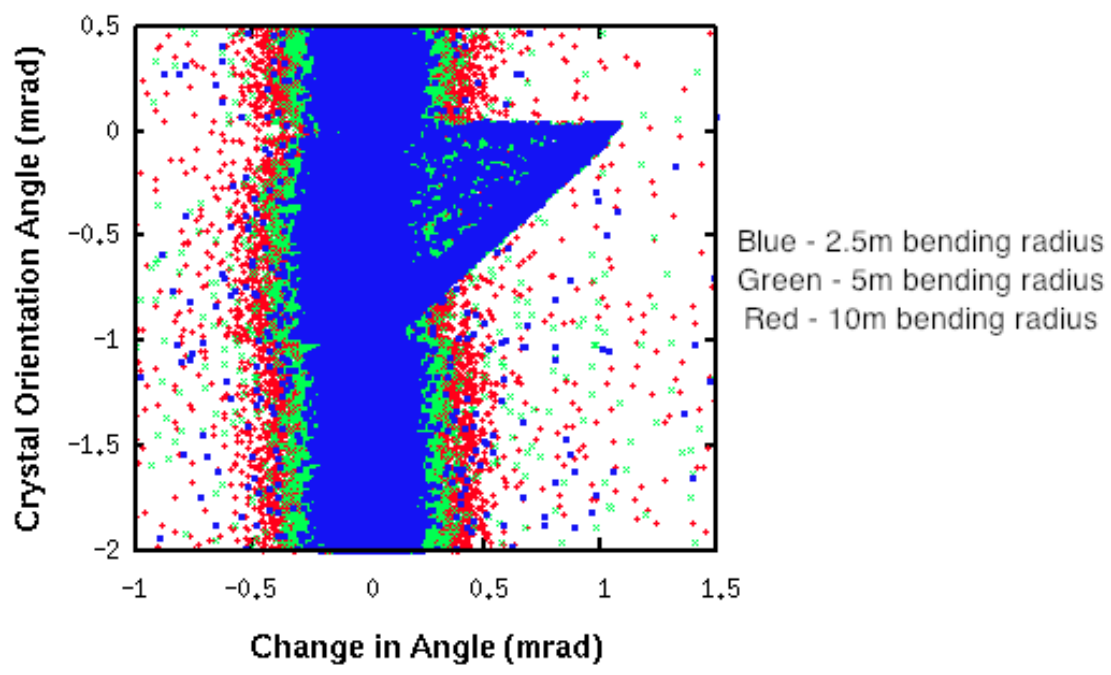

Figure 5: Angular profile with $10 \mathrm{~m} / 10 \mathrm{~mm}, 5 \mathrm{~m} / 5 \mathrm{~mm}, 2.5 \mathrm{~m} / 2.5 \mathrm{~mm}$ bending radius/crystal length. Demonstrates the decrease in multiple scattering as the bending radius decreases.

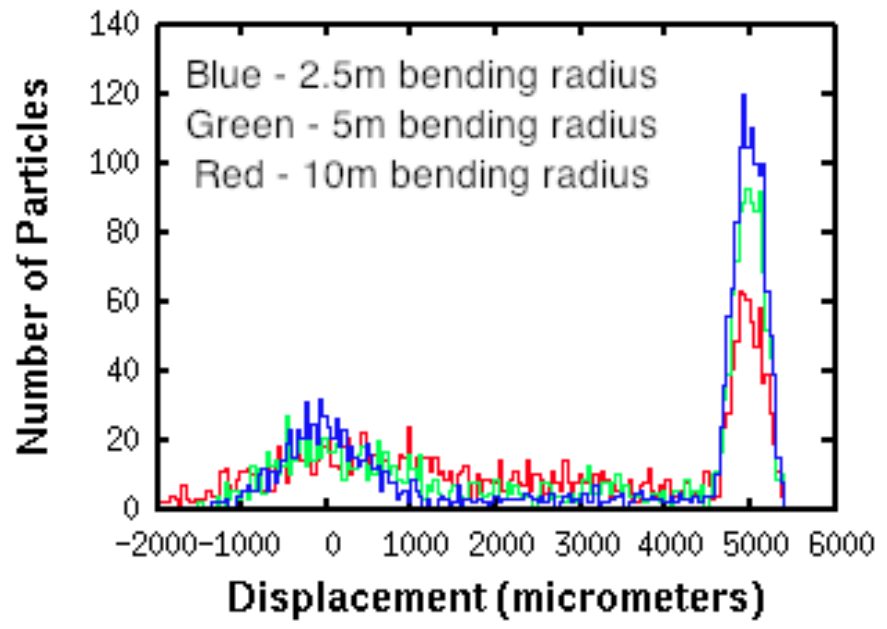

Figure 6: Histogram of the displacement of particles after propagating five meters beyond the crystal in vacuum space. The orientation angle of the crystal is from $-0.051 \mathrm{mrad}$ to $0.049 \mathrm{mrad}$ corresponding to the channeling region. Shown for three different bending radii and crystal lengths. 


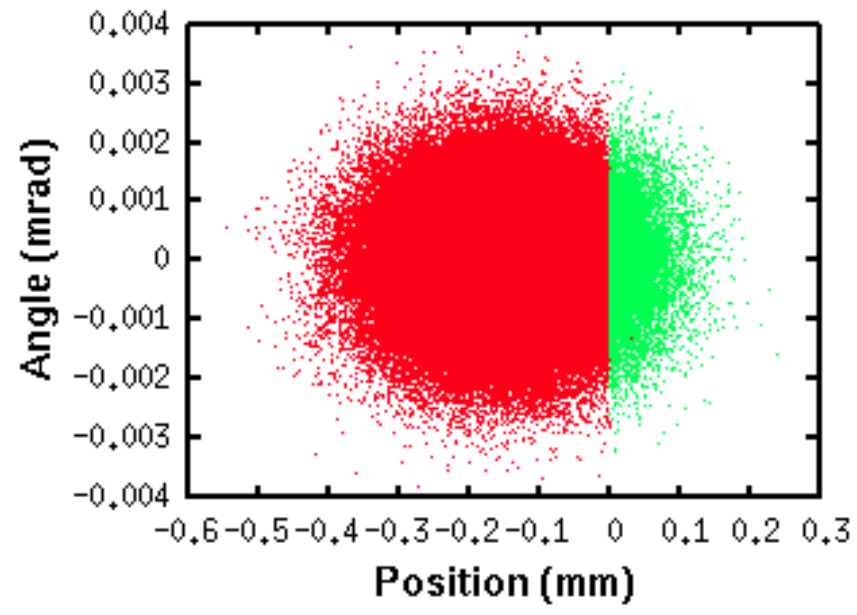

Figure 7: Phase space of angle vs position for the input positron beam. The crystal is placed at position $=0 \mathrm{~mm}$. The beam is offset and only the portion highlighted in green passes through the crystal. The remainder of the beam propagates unaffected.

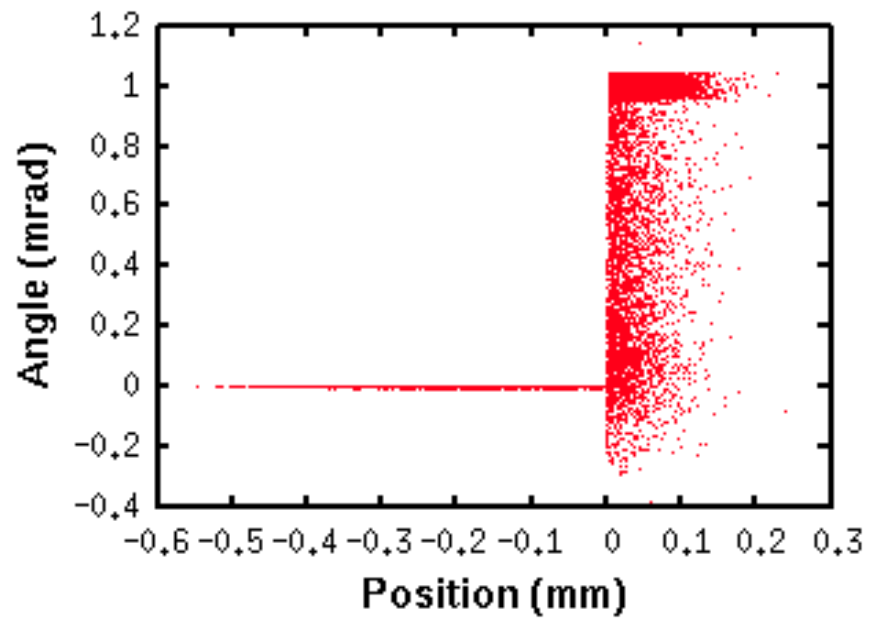

Figure 8: Phase space of angle vs position of positron beam at exit face of the crystal. Note the scale difference from Figure 7. 


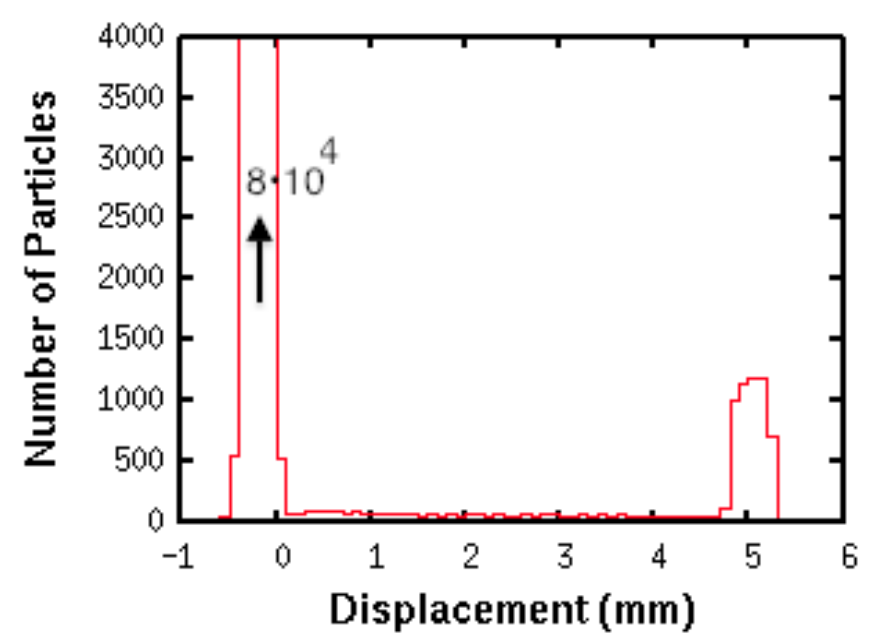

Figure 9: Histogram of the displacement of particles after propagating five meters beyond the crystal in vacuum space with only a portion of the beam passing through the crystal as illustrated in Fig. 7. The majority of the beam is not displaced, the left peak is cut off due to the scale of the graph but reaches its maximum at approximately 80000 particles. The peak on the right represents the channeled particles.

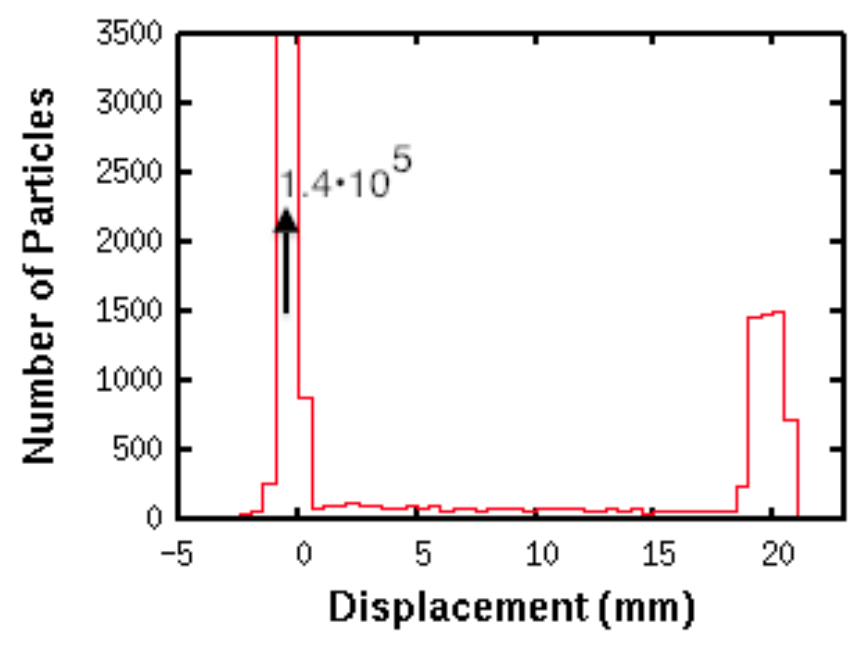

Figure 10: Histogram of the displacement of particles after propagating through a quadrupole magnet after a small portion of the beam passes through the crystal as illustrated in Fig. 7 . Note the scale. The peak on the right represents the particles that were channeled and the remainder of the beam remains unchanged as represented in the peak on the left. 


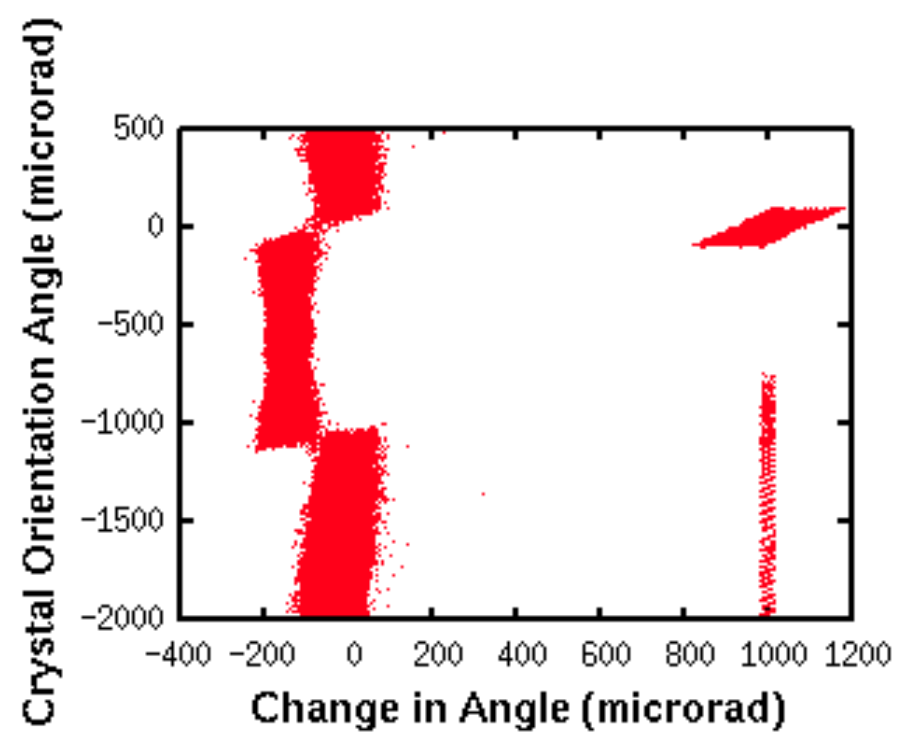

Figure 11: Angular profile of the crystal representing the angular deflection of the particles as a function of the crystal orientation. The crystal is aligned with the beam at an orientation angle of 0 . This plot is the result of the code developed at SLAC. The vertical line parallel to the beam at the change in angle $=1000$ microrad is an artifact of the code dependent on the number of steps. This plot was made using 64000 steps. 


\section{TABLES}

Table 1: FACET Parameters

\begin{tabular}{l|l} 
Energy & $23 \mathrm{GeV}$
\end{tabular}

Emittance $\quad 3 \mu \mathrm{m} \cdot \mathrm{rad}$

Lorentz Factor 45010

Beam Width $\quad 81.6 \mu \mathrm{m}$

Divergence $\quad 0.82 \mu \mathrm{rad}$ 